Editorial

\title{
Role of rituximab in myasthenia gravis' treatment
}

\author{
Volume 6 Issue 3 - 2017
}

\section{Introduction}

Myasthenia gravis (MG) is the most common disorder of neuromuscular transmission with a worldwide prevalence estimated to be between 25 and 142 per million. ${ }^{1} \mathrm{MG}$ is a prototypical autoimmune disease. The muscle acetylcholine receptor (AChR) is the main antigen, as AChR-Ab can be detected in up to $85-90 \%$ of MG patients. AChR-Ab is mainly of IgG1 and IgG3 iso types, and induce severe alterations of the post-synaptic membrane through AChR increased degradation and complement activation. ${ }^{2}$ Around $40 \%$ of patients with AChR-negative MG have serum $\mathrm{Ab}$ to the muscle-specific tyrosine-kinase (MuSK). MuSK-Ab induces severe functional alteration of the neuromuscular junction, although, being mostly IgG4, they do not activate complement. ${ }^{3}$ A small percentage of AChR-negative MG patients have antibodies to lipoprotein-related protein 4 (LRP4), which are also considered pathogenic antibodies. Other antibodies and postsynaptic proteins are still being studied to assess its pathogenic role in the disease. Meanwhile, up to $10 \%$ of MG patients remain antibody-negative MG.

Over the past 40 years, advances in the treatment of MG have notably reduced mortality rates from the disease, as well as patients' quality of life.

Nowadays, attending neurologists have several therapeutic options available to treat MG patients. Control of symptoms can be initially achieved with acetyl cholinesterase inhibitors; however, most patients require immune suppressants (IS) such as corticosteroids, azathioprine, cyclosporine, mycopheno late mofetil, plasma exchange, and intravenous immunoglobulin at some point in their disease course to achieve sustained clinical remission. ${ }^{4}$ Thymectomy, regardless of the presence of a thymoma, is also a therapeutic option in AChRAb positive-MG in light of the recent results of the randomized thymectomy clinical. ${ }^{5}$

Despite these therapeutic options, a subset of patients has medically refractory MG or intolerable adverse effects from medication. Although, there is no standard definition of refractory myasthenia, recently, Sanders et al., ${ }^{6}$ at the International consesus guidancesfor management of myastheniagravis, defined refractory $\mathrm{MG}$ as an unchanged or worse clinical situation after corticosteroids and at least 2 other IS, used in adequate doses for an adequate duration, with persistent symptoms or side effects that limit functioning, as defined by patient and physician.

The exact prevalence of refractory myasthenia is unknown, but it is estimated to occur in approximately $10 \%$ of patients with generalized disease. When patients are deemed to be refractory, more aggressive treatment is warranted to prevent life-threatening crises. Although there are no evidence-based guidelines, agents such as rituximab, high-dose cyclophosphamide, and more recently eculizumab have been used.7 Repeat a more extended thymectomy is another option. The role of bone marrow transplantation is still to be defined; a few case reports have been reported lately. ${ }^{8}$

In the past decades, rituximab (RTX) has been the centrepiece of an increasing number of reports claiming success in managing refractory MG. Rituximab is a chimeric marine-human IgG1 kappa

\author{
Láinez JM,' Ramos-Pachón $\mathrm{A}^{2}$ \\ 'Department of Neurology Service, Clinical University, Spain \\ ${ }^{2}$ Departamen of de Neurology, Catolic University, Spain
}

Correspondence: Láinez JM, departament of Neurology Service, I clinical University, Avda Blasco Ibáñez, 17. 46I,Valencia, Spain, Email Miguel.lainez@sen.es

Received: March 22, 2017 | Published: March 30, 2017

monoclonal antibody that depletes B cells by binding to their CD20 molecule and initiating complement-dependent cytolysis or antibodydependent cell-mediated cytotoxicity. The drug does not impair B cell recovery or antibody production or secretion. Rituximab has been used successfully in a number of autoimmune diseases including systemic lupus erythematosus, autoimmune haemolytic anemia, rheumatoid arthritis, idiopathic thrombocytopenic purpura, mixed cryoglobulinemia, and Wegener granulomatosis. ${ }^{9,10}$

Zaja et al. ${ }^{11}$ first described, in 2000, the benefits of rituximab in refractory myasthenia. Since then several case reports and short series of patients supported this position.

In an early study, Lebrun et al., ${ }^{12}$ prospectively followed up 6 patients ( 3 MuSK Ab positive, 1 AChRAb positive, and 2 sero negative) whose disease remained refractory after thymectomy and treatment with at least 2 conventional agents. Patients received rituximab, $375 \mathrm{mg} / \mathrm{m} 2$ weekly, for 4 weeks, then once a month for 2 months. After the initial phase, treatment was given based on the clinical status. Two patients required repeat infusions for 1 year and 2 others for 2 years. Clinical improvement was noted in all patients within 1 month of treatment. All patients eventually underwent tapering of acetyl cholinesterase inhibitor, prednisone, and other IS therapy. RTX was well tolerated in these patients without significant adverse effects.

Nowak et al., ${ }^{13}$ performed a retrospective study of 14 refractory patients (6 AChRAb positive and 8 MuSK Ab positive) who were successfully treated with rituximab. Rituximab dosing was $375 \mathrm{mg} /$ $\mathrm{m}^{2}$ weekly for 4 weeks with a repeat cycle of $375 \mathrm{mg} / \mathrm{m}^{2}$ weekly for 4 weeks repeated every 6 months. They could reduce prednisone dosage in all patients. There was a statistically significant reduction in the number of plasma exchange sessions after rituximab treatment. AChRAb titers also declined. A clinically evident improvement was sustained in all patients.

A retrospective, observational study performed by Collongues et al. ${ }^{14}$ evaluated the effect of rituximab in both refractory patients (n 13; 
7 AChRAb positive, 3 MuSK Ab positive, 1 both AChRAb and MuSK $\mathrm{Ab}$ positive, and 2 seronegative) and nonrefractory patients dependent on corticosteroids (n 7; 5 AChRAb positive, 1 MuSK Ab posi- tive, and 1 seronegative). All immunosuppressive treatments were stopped before rituximab infusions except for corticosteroids. Rituximab was given according to 2 different protocols: (1) $375 \mathrm{mg} / \mathrm{m}^{2}$ weekly for 4 weeks, and then $375 \mathrm{mg} / \mathrm{m}^{2}$ every 3 months or (2) 2 infusions required if symptoms worsened. Patients were followed for 2 years. Relapses were treated with IVIg or plasma exchange. In the refracory patients, the annualized relapse rate decreased and Myasthenia Gravis Foundation of America (MGFA) classifi- cation dropped. After 1 year, the mean steroid dose in all patients decreased.

In one of the larger studies, Diaz-Manera et al., ${ }^{15}$ reported on long-term follow-up of 17 drug-resistant patients (11 ACh- RAb positive and $6 \mathrm{MuSK} \mathrm{Ab}$ positive) treated with rituximab. They defined drug-resistant patients as those who failed at least 3 secondline agents. Rituximab was given at a dose of $375 \mathrm{mg} / \mathrm{m} 2$ weekly for 4 weeks, then monthly for 2 months; reinfusion were given if symptoms worsened enough to interfere with activities of daily living. Treatment was generally well tolerated. All MuSK Abpositive patients reached minimal manifestation status by 3 months after treatment, and all maintained this favourable status or were in remission at 35 months. Impressively, additional infusions were not needed. Ten of 11 AChRAb-positive patients improved at 3 months. Six patients who required reinfusion did improve, but none reached minimal manifestations status or remission. The average prednisone dose dropped in both groups. There were no significant changes in second-line immunosuppressant dosing in the AChRAb-positive group, unlike the MuSK MG group. MuSK Ab titters declined significantly but the AChRAb titers did not. The authors highlighted the longer lasting treatment effect of rituximab in MuSK Ab-positive patients and hypothesized that this may be due to differences in the pathophysiology of this form of the disease, which is largely mediated by the IgG4 immunoglobulin subclass.

Iorio et al., ${ }^{16}$ reviewed 37 studies with a total of 168 patients. They performed a meta-analysis on 15 studies, excluding case reports and studies including less than 2 patients. The overall response rate to RTX was $83.9 \%$, the response was higher in the MuSK-Ab group but not statistically significant. They did not find any significant correlation between the mean MG severity or the mean number of reinfusion and the response rate. However they found an inverse correlation trend between disease duration and response rate to RTX, although it did not reach the statistical significance. The authors hypothesized this finding may be explained by the selective effect of RTX on the shortlived plasma cells. Only 7/168 patients had adverse effects. They found no generally accepted schedule for RTX application; the most frequent regimen was $375 \mathrm{mg} / \mathrm{m}^{2}$ weekly for 4 weeks.

More recently, Robeson et al., ${ }^{17}$ reported on long-term follow-up of 16 drug-resistant AChRAb positive patients. Rituximab was given at a dose of $375 \mathrm{mg} / \mathrm{m} 2$ weekly for 4 weeks per cycle. The interval between cycles was 6 months. The patients were treated with an initial 2- to 4-cycle regimen. The number of cycles was mainly based on reaching a symptom-free state and patient toleration of tapering or withdrawal of other immunotherapies. A clinical improvement was observed in parallel with complete withdrawal or reduction of other immunotherapies, with all patients achieving complete stable remission, pharmacologic remission, or minimal manifestations based on the MGFA postintervention status criteria. Seven patients (44\%) remained relapse free with a mean follow-up of 47 (range, 18-81) months since the last rituximab treatment. A decrease of AChRAbtiers was seen after every cycle of rituximab treatment. However they found no significant increase in AChRAb titres at the time of relapse, so their role in predicting relapse is less clear. The serum cytokine levels measured were found to be unchanged.

The last International Consensus Guidance for Management of myasthenia gravis6 state the following about Rituximab: "Rituximab evidence of efficacy is building. Formal consensus could not be reached yet." Although in MuSK-Ab MG, they affirm "Rituximab should be considered as an early therapeutic option in patients with MuSK-MG who have an unsatisfactory response to initial immunotherapy".

Nevertheless, in the challenging cases of refractory MG, evidence is emerging that rituximab may be useful, durable and the best tolerated and safest agent. Whereas other options in refractory MG such as maintenance IVIg or PE provide benefits that last only a few weeks per cycle and mandate repeated administration that can stretch for years, 1 or 2 cycles of rituximab appear to produce persistent improvement lasting months or years in MG patients. Across studies, dosing regimens have varied, and observations suggest rituximab may have its most dramatic and durable effect on MuSK antibody-positive MG. Furthermore, RTX has been well tolerated by most patients with MG. Common reported adverse effects are related to infusions and include fever, chills, hypotension, and dyspnea. The development of progressive multifocal leucoxene phalopathy remains a potential risk and was recently reported in 2 patients being treated for refractory MG. ${ }^{18,19}$

Fortunately, a multicenter, placebo- controlled, randomized clinical trial18 about the effect of rituximab on AChR antibodypositive MG has already completed enrolment and it will enlighten us about rituximab position as the go-to intervention for refractory $\mathrm{MG}^{20}$

\section{Acknowledgments}

None.

\section{Conflicts of interest}

None.

\section{Funding}

None.

\section{References}

1. McGrogan A, Sneddon S, de Vries CS. The incidence of myasthenia gravis: a systematic literature review. Neuroepidemiology. 2010;34(3):171-183.

2. Meriggioli MN, Sanders DB. Muscle antibodies in myas- thenia gravis: beyond diagnosis?. Expert Rev Clin Immunol. 2012;8(5):427-438

3. Verschuuren JJ, Huijbers MG, Plomp JJ, et al. Patho- physiology of myasthenia gravis with antibodies to the acetyl- choline receptor, muscle-specific kinase and low-density lipoprotein receptor-related protein 4. Autoimmun Rev. 2013;12(9):918-923

4. Skeie GO, Apostolski S, Evoli A, et al. Guidelines for treatment of autoimmune neuromuscular transmission disorders. Eur J Neurol. 2010;17(7):893-902 is:

5. Wolfe GI, Kaminski HJ, Aban IB, et al. Randomized Trial of Thymectomy in Myasthenia Gravis. N Engl J Med. 2016;375(6):511-522.

6. Sanders DB, Wolfe GI, Benatar M, et al. International consensus guidance for management of myasthenia gravis: Executive summary. Neurology. 2016;87(4):419-425

7. Silvestri, NJ, Wolfe, GI. Treatment-refractory myasthenia gravis. J Clin Neuromuscular Dis. 2014; 15(4):167-178 
8. Bryant A, Atkins H, Pringle CE, et al. Myasthenia gravis treated with autologous hematopoietic stem cell transplantation. JAMA Neurol. 2016;73(6):652-658

9. Gurcan HM, Keskin DB, Stern JN, et al. A review of the current use of rituximab in autoimmune diseases. Int Immunopharmacol. 2009;9(1):10-25

10. Dalakas MC. B cells as therapeutic targets in autoimmune neurological disorders. Nat Clin Pract Neurol. 2008;4(10):557-567.

11. Zaja F, Russo D, Fuga G, et al. Rituximab for myas- thenia developing after bone marrow transplant. Neurology. 2000;55(7):1062-1063.

12. Lebrun C, Bourg V, Tieulie N, et al. Successful treat- ment of refractory myasthenia gravis with rituximab. Eur J Neurol. 2009;16(2):246-250.

13. Nowak RJ, DiCapua DB, Zebardast N, et al. Response of patients with refractory myasthenia gravis to rit- uximab: a retrospective study. Ther Adv Neurol Disord. 2011;4(5):259-266.

14. Collongues N, Casez O, Lacour A, et al. Rituximab in refractory and non-refractory myasthenia: a retro- spective multicenter trial. Muscle Nerve. 2012;46(5):687-691.
15. Diaz Manera J, Martinez Hernandez E, Querol L, et al. Long-lasting treatment effect of rituximab in MuSK myasthenia. Neurology. 2012;78(3):189-193.

16. Iorio R, Damato V, Alboini PE, et al. Efficacy and safety of rituximab for myasthenia gravis: a systematic review and meta-analysis. J Neurol. 2015;262(5):1115-1119.

17. Robeson K, Kumar A, Keung B, et al. Durability of the Rituximab Response in Acetylcholine Receptor Autoantibody-Positive Myasthenia Gravis. JAMA Neurol. 2017;74(1):60-66.

18. Carson KR, Bennett CL. Rituximab and progressive multi-focal leukoencephalopathy: the jury is deliberating. Leuk Lymphoma. 2009;50(3):323-324.

19. Kanth KM, Solorzano GE, Goldman MD. PML in a patient with myasthenia gravis treated with multiple immunosuppressing agents. Neurol Clin Pract. 2016;6(2):e17-e19.

20. A Phase II Trial of Rituximab in Myasthenia Gravis. Clinical Trials.gov NCT02110706. 2016. 